\title{
SPATIAL DISTRIBUTION OF THE SEISMIC HAZARD PARAMETERS IN THE SEISMOGENIC SOURCES OF JAPAN T. M. TSAPANOS ${ }^{1}$, G.CH. KORAVOS ${ }^{1}$, C.I. PATSIA ${ }^{1}$, D.TH. KOULARAS ${ }^{1}$
}

\begin{abstract}
The seismicity parameters in the seismogenic zones of Japan are estimated by the application of two different approaches. These are the maximum likelihood method as well as the Bayesian estimator is invoked in order to check the validity of the results. Both methods allow us to estimate the maximum regional (possible) earthquake $\hat{M}_{\text {max }}$, the well known value $\hat{\beta}$ (b= $\beta$ loge) which is the slope of the magnitude-frequency relation and the mean activity rate $\hat{\lambda}$ of the seismic events. The present study is focused in the first two parameters searching for any pattern on their geographical distribution through the sources of Japan. Two are the main assumptions adopted for both methods: 1) the seismic events are of Poissonian character and 2) the magnitude-frequency law is governed by Gutenberg-Richter type. The methods allow to account the influence of uncertainties of the earthquakes magnitude. Taking into account these properties we found that the values of $\hat{M}_{\max }$ are different and bigger than the observed $M_{\max }^{o b s}$. The estimated b-values show very low values in the seismogenic sources 2 and 7. In comparison with other measurements from other authors these two sources suggested to be areas with very high probability for an large earthquake occurrence.
\end{abstract}

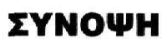

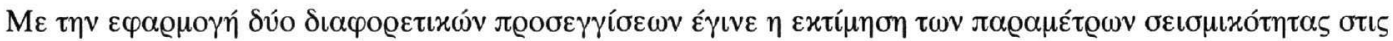

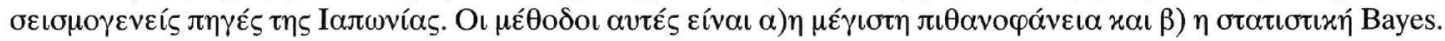

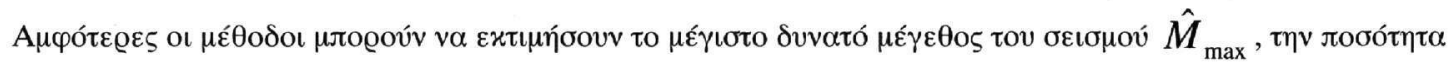

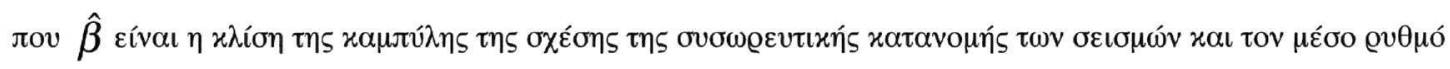

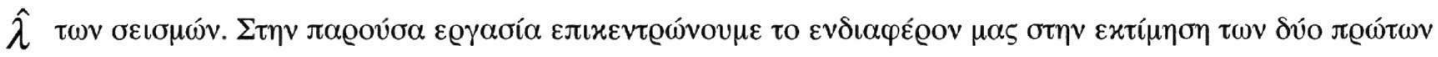

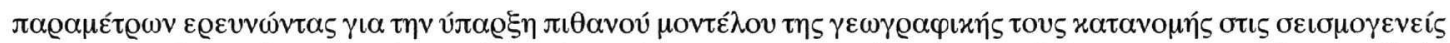

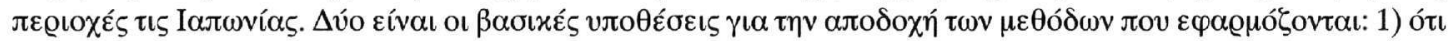

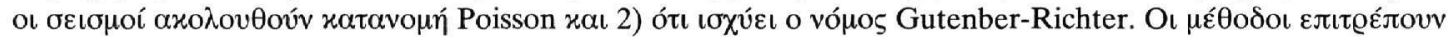

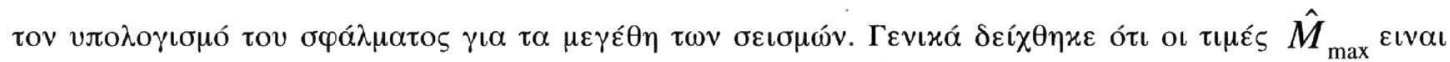

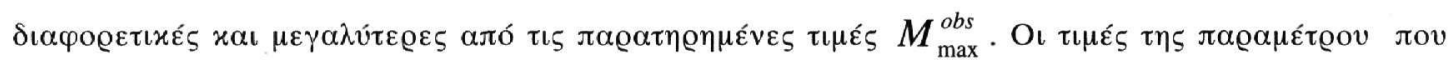

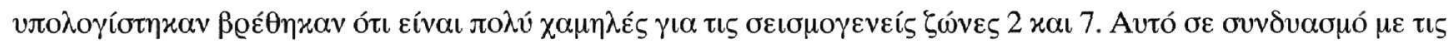

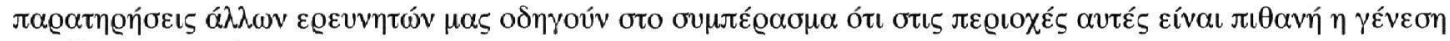

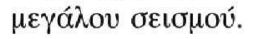

KEY WORDS: seismicity parameters, maximum likelihood, Bayesian approach, maximum possible magnitude, b-values, Japan.

1. Aristotle University of Thessaloniki, School of Geology, Geophysical Laboratory, 54006 Thessaloniki, Greece. 


\section{INTRODUCTION AND DATA USED}

The available catalogues usually contain two different types of information. These consist of: a) the long period (historical) earthquake that is the occurrence over a period of decades or hundreds of years and b) the complete instrumental data for the seismicity of the present century. If we only use the complete (instrumental) data which are available for relatively short periods of time it is difficult to describe a complete seismic active cycle in the seismic hazard analysis.

In order to raise the reliability of the seismic hazard analysis, Kijko and Sellevoll (1989) introduced an alternative method of maximum likelihood, which combines data with different precision (incomplete+complete). The complete data allows the possibility to divide the catalogue into different time intervals of different time lengths, each assume complete above a specific threshold magnitude. The computations of the method are based on assumptions of the Poisson occurrence of main shocks in time with a mean activity rate $\hat{\lambda}$ and the doubly truncated Gutenberg-Richter distribution of earthquake magnitude.

Later Pisarenko and Lyubushin(1999) considered of a Bayesian estimator in order to estimate the seismic hazard parameters in California and Italy. This is a straightforward procedure of estimating the maximum possible magnitude and the related parameters. In order to check the validity of the results obtained by the approach of Kijko and Sellevoll (1989), the Bayesian estimator of Pisarenko and Lyubushin (1998) applied.

The catalogue constructed by Tsapanos et al. (1990) spanning the time-period 1897-1985. This catalogue is updated by taking into account the information up to 1996 from I.S.C. bulletins. This original catalogue is modified in term of magnitudes by considering the magnitudes given by Pacheco and Sykes (1992). Data from 1894 to 1899 are extracted from Abe (1994) as well as from Abe and Noguchi (1983). So the time period covered in the present study is 1894-1996. The time sub-periods for which the catalogue is complete and the corresponding lower threshold magnitudes are: 1894-1996 with $\mathrm{M} \geq 7.0$; $1930-1996$ with $\mathrm{M} \geq 6.5$; $19953-1996$ with $\mathrm{M} \geq 6.0$; and 1966-1996 with $M \geq 5.5$. These are derived on the basis of the cumulative time distribution of the number of earthquakes with magnitudes larger than a certain value. In Figure (1) the data used for the present study, as well as the 11 seimogenic sources in which Japan and the adjacent area is divided by Matsuda (1990), and Papazachos et al. (1997).

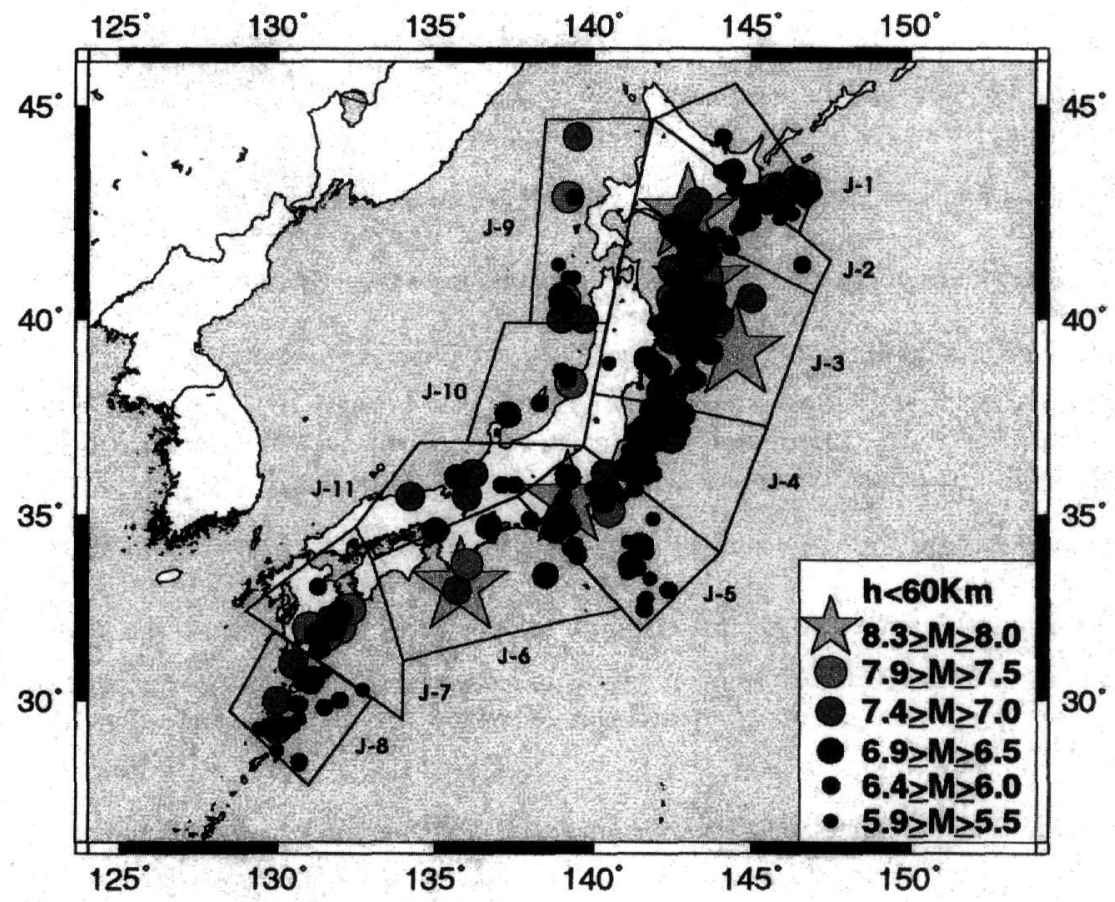

Fig.1. Geographical distribution of the epicenters of the shallow earthquakes and the examined seismogenic sources for the broad area of Japan (modified by Papazachos et. al., 1997). 


\section{THE METHOD APPLIED}

Some basic theoretical considerations are given bellow. We assume the Poisson occurrence of earthquakes with the activity rate 1 and the doubly truncated Gutenberg-Richter distribution $\mathrm{F}(\mathrm{x})$ of earthquake magnitude $\mathrm{x}$. The doubly truncated exponential distribution derived from Page (1968) can be written as:

$$
F(x)=\operatorname{Pr}(X \leq x)=\frac{A_{1}-A(x)}{A_{1}-A_{2}}, \quad m_{\min } \leq x \leq m_{\max }
$$

where

$$
A_{1}=\exp \left(-\beta m_{\min }\right), \quad A_{2}=\exp \left(-\beta m_{\max } \text { and } A(x)=\exp (-\beta x)\right.
$$

while $m_{\max }$ is the maximum regional (possible) magnitude, $m_{\min }$ is the threshold magnitude and $\beta$ is a parameter which is related with $b$-parameter with $b=\beta$ loge. As we referred above we involved only with the instrumental (complete) part of the catalogue. So we can divided the complete catalogue into subcatalogues. Each one has its own time period $\mathrm{Ti}$ and its completeness starting from a known threshold mi $(i=1,2, \ldots, \mathrm{s})$. The values $x_{i j}^{\min }, x_{i j}^{\max }, \mathrm{j}=1,2, \ldots, \mathrm{n}_{\mathrm{i}}$ denote the lower and the upper bounds of the magnitudes. The symbols $\mathrm{s}$ and $\mathrm{n}_{\mathrm{i}}$ are defined as the number of complete subcatalogues and the number of the events in each subcatalogue, respectively. The likelihood function of $q$ for each subcatalogue can be written as a product of two functions:

$$
L_{i}\left(\theta / x_{i}\right)=L_{i \beta} X L_{i \lambda}(2)
$$

where $x_{i}$ denotes the $\left(n_{i} \times 2\right)$ matrix of magnitude values contained in subcatalogue $i(i=1,2, \ldots, s)$.

From the assumption that the earthquake magnitude $\mathrm{x}$ is a random variable distributed according to the doubly truncated Gutenberg-Richter distribution (eqn. 1)it follows that the probability of an earthquake having its magnitude between $x^{\min }$ and $x^{\max }$ is:

$$
p\left(x^{\min }, x^{\max }\right)=\left(A^{\min }-A^{\max }\right) /\left(A_{1 i}-A_{2}\right)
$$

where

$$
A^{\min }=\exp \left(\beta x^{\min }\right), A^{\max }=\exp \left(-\beta x^{\max }\right), A_{2}=\exp \left(-\beta m_{\max }\right) \text {, and } A_{1 i}=\exp \left(-\beta m_{i}\right) \text { for } 1=1, \ldots, \mathrm{s}
$$

Relation (3) makes $\mathrm{L}_{\mathrm{ib}}$ of the form:

$$
L_{i \beta}=\text { const } \prod_{j=1}^{n_{i}} p\left(x_{i j}^{\min }, x_{i j}^{\max }\right)
$$

where const is a normalization factor independent of $\theta$. The assumption that the number of earthquakes per unit time is a Poisson random variable, provides:

$$
L_{i \lambda}=\text { const } \exp \left(-v_{i} T_{i}\right)\left(v_{i} T_{i}\right)^{n_{i}}
$$

where const is the normalizing factor:

$$
v_{i}=\lambda\left[1-F\left(m_{i}\right)\right]
$$

and $\lambda$ is the activity rate corresponding to the threshold magnitude $\mathrm{m}_{\min }<\min (\mathrm{mi}), \mathrm{i}=0, \ldots$,s. This is the mathematical model of the two adopted methods and the equations describe above define the likelihood function of parameters for each complete subcatalogue (see for details in Kijko and Sellevoll, 1989,1992; Pisarenko and Lyubushin, 1999)

\section{RESULTS AND DISCUSSION}

According to Reiter (1990) the maximum regional earthquake magnitude which is the maximum possible earthquake that could occur in a given time interval and tectonic regime and defines an upper bound to earthquake size determined by earthquake processes. This is primarily used in probabilistic analyses.

An effort is made to see if these maximum regional magnitudes are related with the observed ones. In Figure (2) we plotted with black circles the $\mathbf{M}_{\text {max }}$ obtained by the method of Kijko and Sellevoll (1989), while open circles represents the maximum regional magnitude deduced from Pisarenko and Lyubushin (1999). The line of the best fit for the Kijko and Sellevoll (solid line) is of the same slope, as the same line of Pisarenko and Lyubushin 
(dashed line) with equations:

$$
M_{\max }^{K / S}=0.11+1.03 M_{\max }^{o b s}
$$

and

$$
M_{\max }^{P / L}=0.05+1.03 M_{\max }^{o b s}
$$

where the correlation coefficient for these equations are $\mathrm{R}=0.92$ and 0.97 , respectively. This means that the maximum observed and the maximum regional magnitudes are in close correlation, although both maximum regional magnitudes are higher that the observed ones. The mean error of Kijko-Sellevoll's stimates is $0.212 \pm 0.07$, while for Pisarenko-Lyubushin's magnitudes the mean error is $0.178 \pm 0.05$.

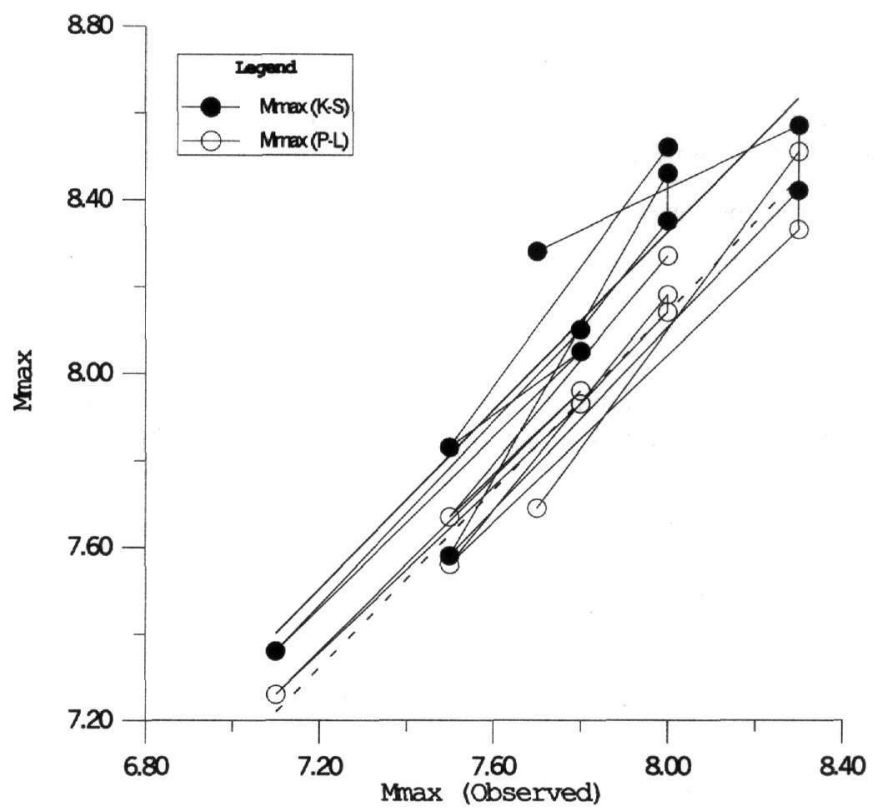

Fig. 2. The maximum regional (possible) magnitude for the eleven seismogenic sources of Japan as it is obtained from Kijko and Sellevoll (1989)- black circles, and by Pisarenko and Lyubushin (1999)- open cicles.

In Figure (3) we have plotted all the available maximum magnitudes along the 11 seismogenic zones in which the area of Japan is divided. Black circles and solid line represent the maximum observed magnitude, while triangles and continues dashed line refer to Kijko-Sellevol results and rhombus and dot-dashed line depict Pisareno-Lyubushin estimates. The first look on this plot shows that the conclusion previously derived, that the maximum regional magnitudes are higher than the observed, is very clear here. Also the magnitudes obtained from Kijko-Sellevoll's method are higher even from those resulted by Pisarenko-Lyubushin's approach. The spatial distribution of all kinds of maximum magnitudes adopted in the present work illustrate low values in the first source but in the next two sources the values are higher with the first minimum in source 4 . Then the magnitude values are higher in source 5,6,7, while we observed the second minimum in source 8 . In source 9 we obtained a maximum value and in 10 we see the last (third) minimum. In the last source (11)high values of maximum regional magnitudes demonstrated. We want to notice here that the seismicity of source 4 for the present century is one of the lowers in the examined area But if we took into account the large shock with magnitude $M_{\max }^{o b s}=8.0$ (Usami, 1996) which occurred in 1677 the maximum regional magnitude changed in: $\mathrm{M}_{\max }=8.28 \pm 0.26$ (kijko-Sellevoll) and $\mathrm{M}_{\max }=8.12 \pm 0.13$ (Pisarenko-Lyubushin. The advantage of the methods used is revealed clearly in this example, otherwise this information will be lost and the seismicity estimation may be underestimated. 


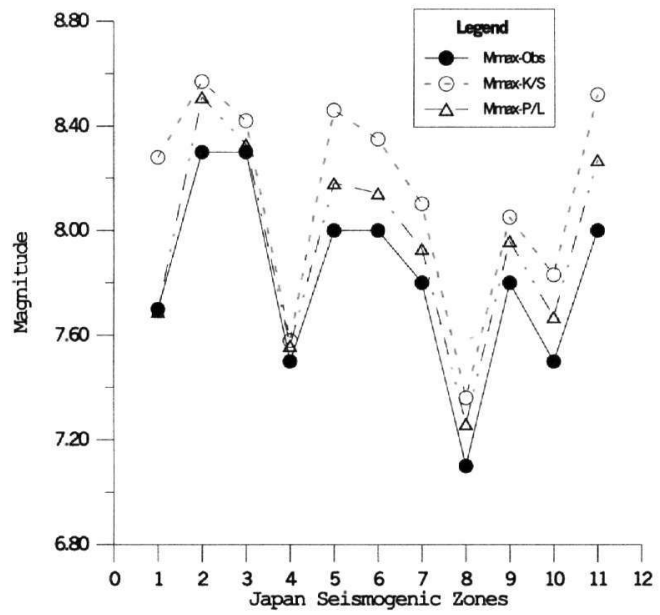

Fig. 3. The maximum magnitude for the 11 seismogenic sources of Japan: observed is represented by black circles; regional (possible) is depicted by triangles (Kijko-Sellevoll's approach), while by open circles this magnitude obtained by Pisarenko-Lyubushin's method is illustrated.

Tsapanos (2000) applied Kijko-Sellevoll's approach and found for the whole Japan that $\mathrm{M}_{\max }=8.69 \pm 0.60$, while the estimated $b$-value $=0.92 \pm 0,02$. Interesting illustration is observed in the spatial distribution of the $b$ values (fig. 4) of Gutenberg-Richter relation, along the 11 seismogenic sources of Japan. These values are calculated from the estimated values of the seismic hazard parameter $\beta$. In this figure we defined by dark circle the $b$ value derived from Kijko-Sellevoll's method and with open triangle we present the b-values obtained by PisarenkoLyubushin's approach. Anomalously low b-values observed in sources 2 and 7 and this is inspected in the results deduced by both methods applied. The value for source 2 of $b=0.51 \pm 0.14$ (Kijko-Sellevoll) and $b=0.53 \pm 0.11$ (Pisarenko-Lyubushin). The estimated $b$-value $=0.58 \pm 0.13$ for source 7 (Kijko-Sellevoll) and $b$-value $=0.50 \pm 0.12$ (Pisarenko-Lyubushin). Results deduced from inversion analysis of GPS data for northeast Japan (Miyazaki et al ., 1998) show that the subducting velocity of the Pacific plate off Hokkaido deduced from the plate motion model is $7.8 \mathrm{~cm} / \mathrm{yr}$. Based on these measurements and on the results derived by his team Ito et al. (2000) suggested a strong interplate coupling which indicates strain accumulation for the next great interplate earthquake. This place is coincide with the source 2 . The low b-values found in the present study support the previous results, for the next great interplate earthquake, obtained by different methods and different authors. Also Papazachos et al. (1994) defined for this zone high probability for an earthquake occurrence with magnitude $M \geq 7.5$ during the period 1993-2002.

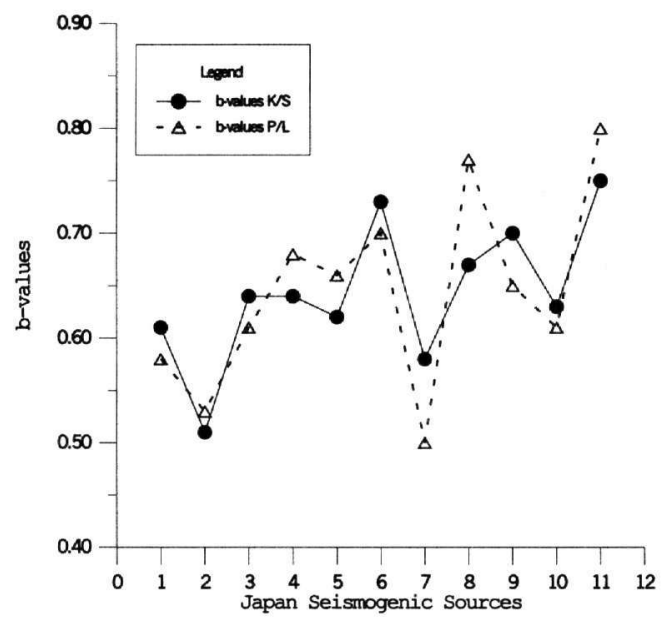

Fig. 4. The b-values for the 11 seismogenic sources of Japan: black circles represent the values obtained by KijkoSellevoll's method, while the open triangles depict the values taken from Pisarenko-Lyubushin's approach. 
The next very low b-values are observed in source 7. The coupling in this region is estimated (El-Fiky et al., 1999)as a very strong (95\%)one. The distribution of interseismic slip rate on the plate boundary between the subducting Philippine sea plate and the continental plate in the southwest Japan is computed (Ito et al., 1999) based on the displacement rates obtained from the an inversion analysis for continuous GPS data. The obtained results from this research suggested that the deformation of this area does not behave like an elastic body but a rigid body. Large crustal deformation in the area of source 7, were also estimated by Hashimoto and Jackson (1993). All these measurements with the very low b-values estimated can lead us to the conclusion that this source is of high risk for a large earthquake occurrence. Papazachos et al. (1994) estimated for this zone a probability of 0.46 for an earthquake occurrence with magnitude $\mathbf{M}=7.8$ during the period 1993-2002.

\section{CONCLUSIONS}

In the present study we apply two approaches in order to estimate the seismic hazard parameters in 11 seismogenic sources of Japan. The estimated parameters are the $\mathbf{M}_{\max }$ which is the maximum regional (possible) magnitude and the $b$-value obtained through the relationship $b=\beta$ loge. The magnitudes $M_{\max }$ obtained through Kijko and Sellevoll (1989) and Pisarenko and Lyubushin (1999) compared with the corresponding observed $M_{\max }$ and both found to be larger that the observed ones. The largest is the one obtained by Kijko and Sellevoll's approach. Special interest is given to the spatial distribution of the b-values. Significantly low b-values are estimated for the seismogenic sources 2 and 7 which in comparison with other measurements from different authors are suggested as zones of high risk and is concluded that both are places for the next large interplate earthquake.

\section{REFERENCES}

ABE, K. (1994). Instrumental magnitudes of historical earthquakes, 1892 to 1898, Bull. Seismol. Soc. Am., 84, 415-425.

ABE, K. and NOGUCHI, S. (1983). Determination of magnitude for large shallow earthquakes 1898-1917, Phys. Earth Plan. Inter., 32, 45-59.

EL-FIKY, G.S., KATO, T. and OWARE, E.N. (1999). Crustal deformation and interplate coupling in the Shikoku district, Japan, as seen from continuous GPS observation, Tectonophysics, 314, 387-399.

HASHIMOTO, M. and JACKSON, D. (1993). Plate tectonics and crustal deformation around the Japanese islands, J. Geophys. Res., 98, 16149-16166.

ITO, T., YOSHIOKA, S. and MIYAZAKI, S. (1999). Interplate coupling in southwest Japan deduced from inversion analysis of GPS data, Phys.Earth Planet. Inter., 115, 17-34.

ITO, T., YOSHIOKA, S. and MIYAZAKI, S. (2000). Interplate coupling in northeast Japan deduced from inversion analysis of GPS data, Earth Planet. Scie. Lett., 176, 117-130.

KIJKO, A. and SELLEVOLL, M.A. (1989). Estimation of earthquake hazard parameters from incomplete data files. Part I: Utilization of extreme and complete catalogues with different threshold magnitudes, Bull. Seismol. Soc. Am., 79, 645-654.

KIJKO, A. and SELEVOLL, M.A. (1992). Estimation of earthquake hazard parameters from incomplete data files. Part II: Incorporation of magnitude heterogeneity, Bull. Seismol. Soc. Am., 82, 120-134.

MATSUDA, T. (1990). Seismic zoning map of Japanese islands, with maximum magnitudes derived from active fault data, Bull. Earthq. Res. Inst., Univ. of Tokyo, 65, 289-314.

MIYAZAKI, S., TADA, T., SAGIYA, T., DONG, D. and JOHNSON, J.(1998). Regional crustal deformation of Japan derived by Japanese national GPS array, EOS Trans. AGU 79 (45), FI86, fall meet. Suppl.

PACHECO, J.F. and SYKES, L.R. (1992). Seismic moment catalog of large shallow earthquakes, 1900 to 1989 , Bull. Seismol. Soc. Am., 82, 1306-1349.

PAGE, K. (1968). Aftershocks and microaftershocks of the great Alaska earthquake of 1964, Bull. Seismol. Soc. Am. 58, 1131-1168.

PAPAZACHOS, B.C., PAPADIMITRIOU, E.E., KARAKAISIS, G.E. and TSAPANOS, T.M. (1994). An application of the time and magnitude predictable model for the long term prediction of strong shallow earthquakes in the Japan area, Bull. Seismol. Soc. Am., 84, 426-437.

PAPAZACHOS, B.C, PAPADIMITRIOU, E.E, KARAKAISIS, G.F. and PANAGIOTOPOULOS, D.G. (1997). Long-term Earthquake prediction in the circum-Pacific convergent belt, Pageoph, 149, 173-217.

PISARENKO, V.F. and LYUBUSHIN, A.A. (1999). A Bayesian approach to seismic hazard estimation: Maxi- 
mum values of magnitudes and peak ground accelerations, Earthq. Research in China (English Edition), Vol. 13 , (in press).

REITER, L. (1990). Earthquake hazard analysis, Columbia Univ. Press, New York, 254 pp.

TSAPANOS, T.M. (2000). Evaluation of the seismic hazard parameters for selected regions of the world: The maximum regional magnitude, Ann. di Geof., (in press)

TSAPANOS, T.M., SCORDILIS, E.M., and PAPAZACHOS, B.C. (1990). A global catalogue of strong earthquakes, Publ. Geophys. Lab. Univ. Thessaloniki, Thessaloniki, 9, 90pp.

USAMI, T. (1996). Materials for comprehensive list of destructive earthquakes in Japan, 416-1995 (revised and enlarged edition), Univ. of Tokyo Press. 